\title{
Efficient in-game communication in collaborative online multiplayer games
}

\author{
Fotios Spyridonis, Damon Daylamani-Zad, Margarita P. O'Brien \\ Department of Computing and Information Systems \\ University of Greenwich \\ London, United Kingdom \\ \{f.spyridonis, d.d.zad, om343\}@greenwich.ac.uk
}

\begin{abstract}
The ability to communicate during online gaming is one that has become fundamental. Players could use voice, textual chat, pre-determined commands or a combination of these methods in order to command a team, follow an appointed leader or simply to socialise with friends. There is an abundance of reasons why a person will choose to utilise the conversational frameworks available, however, when considering a game where players must work together in order to reach a common goal, it is up for interpretation to determine which method works most efficiently. The aim of this work is to address this need through a game that utilises these three methods of communication in order to determine their efficiency. A combination of qualitative and quantitative methods was employed to formulate a well-rounded conclusion. Results indicated that a combination of voice and predetermined commands is the most efficient method of in-game communication in online, task-oriented games.
\end{abstract}

Keywords-Games; Design; Gameplay experience; Communication; Communicative Action Theory

\section{INTRODUCTION}

In the world of Massively Multiplayer Online Games (MMOGs) communication between players is essential. There is an abundance of methods that are typically employed in such environments ranging from verbal to non-verbal forms of interaction. Choosing therefore an appropriate communication model can and often provides a positive player experience. In principle, MMOGs use different player-to-player communication forms based on their style of gameplay and the level of immersion required to fully enjoy the experience of a game. Typically, this can be achieved through voice, textual chat, or simple pre-determined commands [1]. However, not all or only one of these methods of communication will work for all games and genres. There are many different factors that can influence which form of communication is the best to use. Past research has indicated that voice communication is more effective in games where players knew each other prior to play [2]. As a result, textual chat is preferred as a means to communicate in situations where players do not personally know each other [3]. Additionally, Lober et al. [4] identified that textual chat is a more effective form of communication with larger team sizes in different settings, but voice seemed to be faster when teams had to make quick decisions [5]. However, there is little research on what is the more preferred and efficient form of communication in task-oriented MMOGs.

In order to assess which communication form is optimal to use, this paper will investigate three common communication forms - voice, textual chat and pre-determined commands - with an aim to propose an efficient communication model that could be used when designing new team-based, task-oriented games. For this study, we adopt the definition of efficiency by [6] as the resources used (e.g. time, effort) in relation to the results achieved. A puzzle game is used to assess the above forms of communication, as this game genre is mainly focused on achieving a goal such as the successful completion of a task or quest [7], which is in line with the aim of this paper.

\section{BACKGROUND}

\section{A. Communication in Games}

Communication in video games has extensively been studied in the past. Most of previous work was concerned with the psychological effects of games [8]. Anderson and Bushman [9] and Sherry [10] presented meta-analyses of the literature about aggression and arousal in video games. Similarly, communication in video games was studied in disciplines such as cognitive skills development [11], health [12] and education [13]. However, the majority of previous work looked at video games as a means to improve and enhance communication. To the best of the authors' knowledge, there's little research that has explored player communication within video games and what constitutes efficient collaboration among players during gameplay. In order to address this gap, the Communicative Action Theory (CAT) that was originally coined by Habermas [14] will be used as a framework, as it allows to analyse and describe interaction in various settings. CAT has been extensively applied to the development of Information Systems [15] and the quality of process [16], amongst other disciplines. Little work, if any, has looked at games through the lenses of communicative action.

\section{B. Communicative Action Theory in Games}

Manninen [1] outlined six main types of communicative action that are found in a conversation in online games. These have been further organised into positive, negative or neutral according to their hypothesised behaviour:

i. Dramaturgical. Presentation of a player in a public forum. (Neutral)

ii. Instrumental. A player aiming to advance his or her personal interests. (Positive)

iii. Normatively regulated. Players in a social group act in accordance with common values. (Positive) 
iv. Strategic. Two or more players aiming to bring about a desired state of affairs. (Positive)

v. Communicative. Players coordinate and arrive at a common consensus of a situation. (Neutral)

vi. Discursive. Entails the explanation, discussion and even criticism of the validity norms that govern communicative action. (Negative)

It is anticipated that strategic and discursive actions will be more prevalent as more than one players will be involved in the proposed game.

\section{MAZE GAME PROTOTYPE}

The prototype was developed using Unity and UNET for the in-built networking facility that would allow for evaluating our prototype as an online game. The main objective of the game is to escape a simple maze (Fig. 1) that the players have the option to either complete together or separately (Stage 1). In doing so, the players have to avoid obstacles that have been placed within the maze in order to result in positive e.g. instrumental or strategic actions dominating the communication between the players, as opposed to allowing players to act as wished, which may allow for other actions to arise e.g. discursive actions. An early ladder toggle and sphere collection were further implemented within the maze game (Stage 2) in order to facilitate cooperation and teamwork.

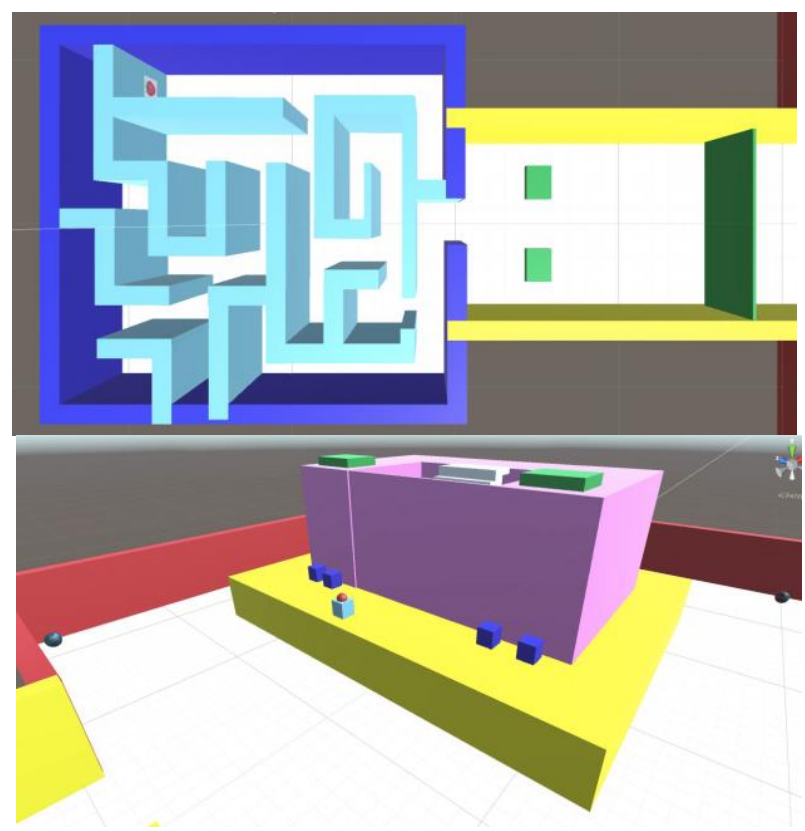

Fig. 1. Maze level design

\section{A. Communication forms in Stage 1}

Instrumental actions are expected to be seen in Stage 1 more prolifically. In a situation where the end goal is not in a known location and neither player is familiar with their surroundings, it is common for one player to take charge. Specifically, if a player has previous gaming experience, then s/he would give instructions that their partner would follow, resulting in instrumental communicative actions taking place. Dramaturgical actions could also be present as a result of players taking on leader/follower roles. The players may want to portray a positive image of themselves on their partner in order to justify their role. Communicative and normatively regulated actions could also become apparent once a subconsciously agreed hierarchy has been attained.

\section{B. Communication forms in Stage 2}

Strategic or instrumental actions will be more evident in the ladder toggle stage, as it allows players to speak to each other, either to tell their partner the solution if they have discovered it or to converse about possible courses of action. However, this stage is possibly the most likely to cause discursive actions. For instance, if a player is leaving their partner to collect the spheres alone, it could cause friction and criticism of each other's actions. On the contrary, if players could work well together, this could result in positive actions such as communicative or normatively regulated in the form of praising.

\section{Gameplay flow}

The behaviour players may demonstrate is mapped to the gameplay flow for Stage 1 (Fig. 2) and Stage 2 (Fig. 3). It is anticipated that players who use pre-determined commands are more likely to take the negative routes due to inability to communicate their exact location. On the other hand, using textual chat or voice could give more lexical freedom and help take positive routes; however, this could result in negative communicative actions. The red areas demonstrate instances where discursive actions could become prevalent e.g. when a player demonstrates selfish behaviour.

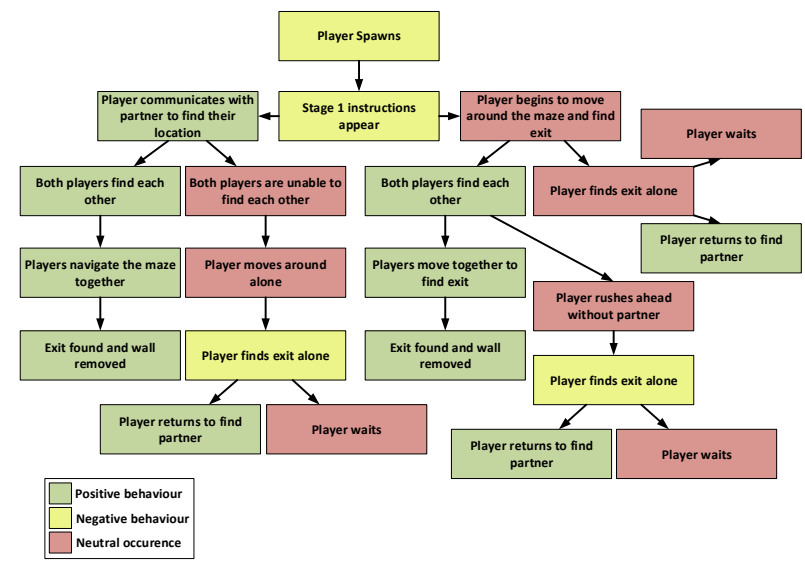

Fig. 2. Maze gameplay flow - Stage 1

\section{EVALUATION}

Accordingly, the hypothesis being tested is that communication forms that exhibit the most positive behaviour will be preferred and will be the most efficient amongst players, while at the same time any negative behaviour will be reduced.

\section{A. Methodology}

Twelve undergraduate game design students from the University of Greenwich (4 female; 8 male, 18-25 years old) took part in the tests. They all had previous experience playing video games on a range of platforms. Recruitment was through a call for participation to evaluate our game. The study was approved by the University of Greenwich Research Ethics Committee and informed consent was obtained from all 
participants. Participants were asked to complete two main tasks (T) that were designed to assess efficiency through a form of communication outlined earlier; [T1] navigate through the maze level and escape the maze (Stage 1), and [T2] reach the top of the tower and escape (Stage 2). Participants were randomly allocated in pairs in order to assess their preferred form of communication. They were then asked to play through each stage and complete the aforementioned tasks in the quickest time possible using their allocated form of communication. Both qualitative and quantitative data i.e. time taken to complete each stage, screen recordings of each play through, and participants' opinions using the think-aloud protocol were recorded and analysed, in order to determine which form of communication has been the most efficient. A post-test survey was also administered to collate the participants' opinions about their allocated forms of communication. In order to analyse the results, the six main types of communicative action were used to annotate the conversation scripts in order to determine the efficiency of the communication.

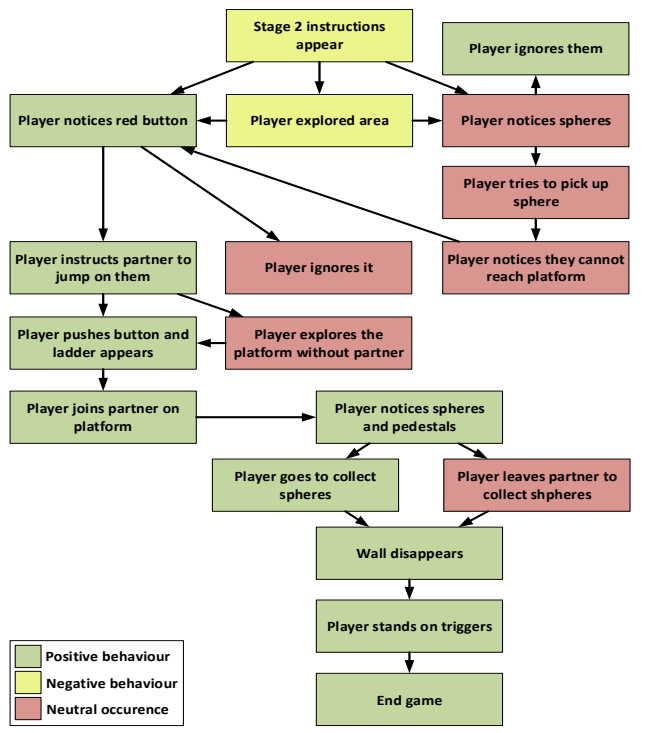

Fig. 3. Maze gameplay flow - Stage 2

\section{B. Results}

This section presents the results in terms of occurrence of each communicative action, prevalence per form of communication, the post-test analysis, and completion time.

\section{1) CAT action occurrence}

Positive behaviour in terms of strategic and instrumental actions was rife in the participants' conversations. However, negative behaviour in the form of discursive actions began to appear in the voice and textual conversations at the end of the test. As such, we decided to divide the discursive actions into two sub categories of disruptive and non-disruptive. We define the non-disruptive category as comments about the game that are not goal oriented, but are still about the experience, the environment and setting e.g. criticisms, observations or reflections. For example, a participant commented on another participant's gameplay with "I feel like you are not right clicking" or another participant made the following comment: "I like the colour of the spheres...". The disruptive category is defined as conversations that were not relevant to the game, the test or the task at hand, and which disrupt the flow. These could range from real-world comments about real life, hobbies and interests to possible inappropriate comments.

\section{2) CAT action per form of communication}

Fig. 4 displays how often each communicative action was present in the voice and textual chat tests. As predicted, the most common actions present in voice chat were strategic and discursive i.e. both positive and negative behaviours. This is unsurprising as the nature of the product relies on players using strategic actions in order to complete the game. Furthermore, the nature of voice chat increases the chances of discursive actions being present due to the issue of freely being able to speak at any time. Strategic actions (positive behaviour) occurred the most in textual chat. Due to the limitations that are present with textual chat functionality in games, such as the requirement to stop movement whilst typing a message, the participants would have to be more economical with the phrases they choose. Furthermore, the instances of disruptive discursive chat were greatly reduced - there was only one occurrence as opposed to the 12 occurrences during the voice chat test. This observation rules in favour of textual chat. These limitations greatly decrease the communicative actions identified as negative and promote more productive communicative actions, which in terms of goaloriented games is highly beneficial.

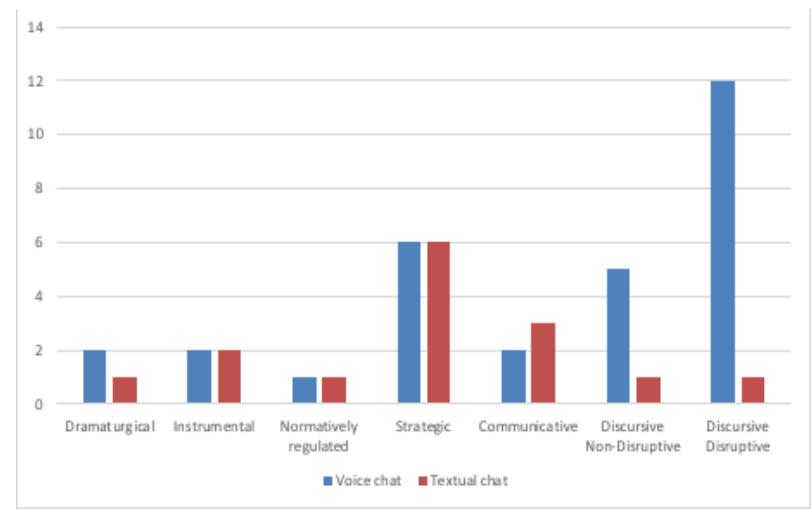

Fig. 4. Average communicative actions in voice vs. textual chat tests

\section{3) Post test analysis}

Participants were further asked to rate the method of communication on a Likert scale of 1-10. Voice chat was the highest rated method (mean=9, $\mathrm{SD}=0.63$ ), whilst predetermined commands was found the least popular (mean $=3.5$, $\mathrm{SD}=0.76$ ) despite the positive behaviour that was exhibited by its use. Textual chat had a mean $=4.5$ with $\mathrm{SD}=1.38$. It is clear that participants found the method with more constraints as less efficient. When answering the post-test survey, participants stated that they felt voice chat was an efficient method of communication for the tasks presented to them despite the occurrence of negative behaviour. One participant stated that using textual chat was "quick", which can be considered positive. However, most participants found this method to be inefficient for the reason that one had to stop moving in order to communicate with their partner. This can be considered a fundamental flaw with the nature of textual chat, as it is arguably the slowest of the three methods being tested. On the other hand, all participants agreed on the efficiency of pre-determined 
commands. They expressed that they would prefer to use a wider range of commands. One participant stated that the use of predetermined commands adds a new dimension of difficulty to games. It disallows the players from being too "chatty" and allows for more focus on the game, adding to the sense of immersion players feel.

\section{4) Completion time}

The time taken for the pre-determined command tests were faster than the other two forms of communication despite being the most restrictive (Fig. 5). A possible reason would be because using voice or textual chat allows players to speak to each other more freely which results in unhelpful chat. Furthermore, it can be noted that textual chat participants never had the fastest time when completing a task. This matches comments that when communication was needed, players had to stop moving in order to type a message, which slowed down the players' progress. As a result, textual chat was not the most efficient method of communication in terms of completing an in-game goal. Finally, participants using pre-determined commands completed all the tasks in the fastest time (mean $\mathrm{t}=06: 44, \mathrm{SD}=0.05$ ), and textual chat was again the slowest (mean $\mathrm{t}=08: 45, \mathrm{SD}=0.02)$. Voice chat had a particularly average time (mean $\mathrm{t}=07: 02, \mathrm{SD}=0.04$ ) when considering both times taken for specific tasks, and overall, this indicates that voice chat is an efficient method of communication in the situation presented; however as mentioned previously, it can be disadvantageous when considering the disruptive actions that are taken during voice communications.

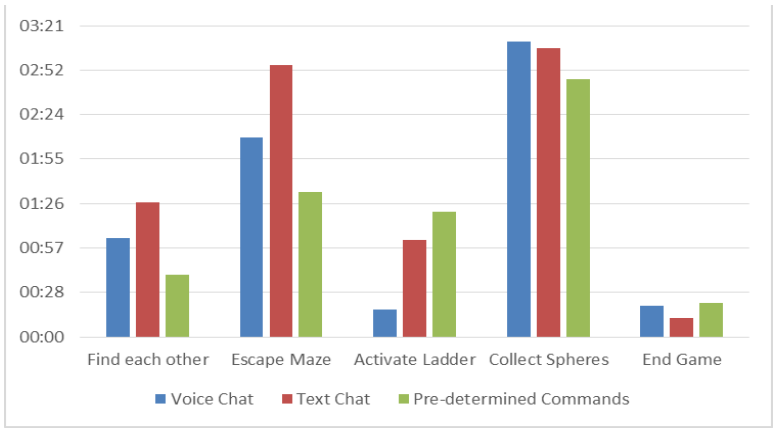

Fig. 5. Average time for completing each task

\section{CONCLUDING DISCUSSION}

In this paper we investigated the efficiency and preferred form of communication in team-based, task-oriented MMOGs. Our results indicated that voice was rated the highest amongst participants, whereas textual chat and pre-determined commands had the most strategic actions present and the fastest completion time, respectively. While voice produced a significant amount of discursive actions, the participants gave it very high ratings, praising its efficiency, but it did not present the slowest completion time despite an overwhelming amount of unhelpful chat. In contrast, pre-determined commands had no discursive actions due to participants being unable to speak freely, as well as the fastest completion time, presumably due to their inability to formulate their own messages. Regardless, pre-determined commands received the lowest rating. Lastly, textual chat had the slowest completion time of all three methods and a relatively low average score, barely coming above pre-determined commands despite giving more freedom of speech. A redeeming quality of textual chat is that it significantly reduced the number of discursive action incidents and unhelpful chat when compared to voice. Our findings show that there was no singular, optimal method of in-game communication in team-based tasks. It is suggested that two (or more) forms of communication could be combined - voice and pre-determined commands - which supports previous findings that a game should offer several processes of collaboration [17]. On the other hand, textual chat was found that can hinder the smooth playthrough of a game, especially when a goal needs to be met. Future work will investigate the addition of more tasks and how players deal with different situations. Overall, this work can contribute in ongoing efforts to optimise in-game communication in online multiplayer games.

\section{REFERENCES}

[1] T. Manninen, "Interaction Forms and Communicative Actions in Multiplayer Games,” Int. J. Comput. game Res., vol. 3, no. 1, 2003.

[2] J. Halloran, G. Fitzpatrick, Y. Rogers, and P. Marshall, "Does it matter if you don't know who's talking?: multiplayer gaming with voiceover IP," in CHI '04 extended abstracts on Human factors in computing systems SE - CHI EA '04, 2004, pp. 1215-1218.

[3] K. Hew, M. Gibbs, and G. Wadley, "Usability and sociability of the Xbox Live voice channel," in Pisan, Y.: Proceedings Australian Workshop on Interactive Entertainment (IE2004), Creative and Cognitive Studios Press, Sydney, 2004, pp. 51-58.

[4] A. Löber, G. Schwabe, and S. Grimm, "Audio vs. chat: The effects of group size on media choice," in Proceedings of the Annual Hawaii International Conference on System Sciences, 2007, pp. 41-41.

[5] A. Löber, S. Grimm, and G. Schwabe, "Audio vs chat: Can media speed explain the differences in productivity?," ECIS, no. 2006, 2006. ISO, "ISO 9241-11:2018(en), Ergonomics of human-system interaction - Part 11: Usability: Definitions and concepts," 2018. [Online]. Available: https://www.iso.org/obp/ui/\#iso:std:iso:9241:11:ed-2:v1:en. [Accessed: 17-Jun-2018].

[7] M. J. P. Wolf, The medium of the video game. University of Texas Press, 2002.

[8] J. Federman, S. Carbone, H. Chen, and W. Munn, The social effects of electronic interactive games: An annotated bibliography. Studio City, CA : Mediascope, Inc, 1996.

[9] B. C. A. Anderson and B. J. Bushman, "Effects of Violent Video Games on Aggressive Bheaviour, Aggressive Cognition, Aggressive Affect, Physiological Arousal and Prosocial Behavior: A MetaAnalytic Review of the Scientific Literature," J. Psychol. Sci., vol. 12, no. 5, pp. 353-359, 2015.

[10] J. Sherry, "The effects of violent video games on aggression..," Hum. Commun. Res., vol. 27, no. 3, pp. 409-431, Jul. 2001.

[11] C. S. Green and D. Bavelier, "Learning, attentional control, and action video games," Current Biology, vol. 22, no. 6. NIH Public Access, pp. R197-206, 20-Mar-2012.

[12] P. M. Kato, "Video Games in Health Care: Closing the Gap," Rev. Gen. Psychol., vol. 14, no. 2, pp. 113-121, 2010.

[13] J. J. Vogel, D. S. Vogel, J. Cannon-Bowers, C. A. Bowers, K. Muse, and M. Wright, "Computer Gaming and Interactive Simulations for Learning: A Meta-Analysis," J. Educ. Comput. Res., vol. 34, no. 3, pp. 229-243, Apr. 2006.

[14] J. Habermas, "The theory of communicative action." Beacon Press, 1984.

[15] D. Cecez-kecmanovic and M. Janson, "Communicative Action Theory: An Approach to Understanding the Application of Information Systems," pp. 183-195, 1999.

[16] F. Murtagh, M. Pianosi, and R. Bull, "Semantic mapping of discourse and activity, using Habermas's theory of communicative action to analyze process," Qual. Quant., vol. 50, no. 4, pp. 1675-1694, Jul. 2016.

[17] R. Hämäläinen, T. Manninen, S. Järvelä, and P. Häkkinen, "Learning to collaborate: Designing collaboration in a 3-D game environment," Internet High. Educ., vol. 9, no. 1, pp. 47-61, 2006. 